\title{
Escolha dos sons-alvo para terapia: análise com enfoque em traços distintivos
}

\author{
Choice of target sounds for therapy: analysis focused on \\ distinctive features
}

\author{
Marizete Ilha Ceron¹, Márcia Keske-Soares², Giovana Ferreira Gonçalves ${ }^{3}$
}

\begin{abstract}
RESUMO
Os alvos de tratamento podem dificultar ou facilitar as aquisições no inventário fonológico. Este trabalho tem como objetivo verificar a eficácia da proposta de terapia de Oposições Múltiplas, considerando a escolha dos sons-alvo, e analisar a aquisição de sons no inventário fonológico e os traços distintivos, enfocados a partir dos sons-alvo. O grupo pesquisado constituiu-se de cinco crianças com desvio fonológico, média de idade de 6;1 (anos;meses). Foram realizadas avaliações fonoaudiológicas e complementares. Após as avaliações, os sujeitos foram submetidos à fonoterapia pelo Modelo de Oposições Múltiplas. Depois do tratamento, realizou-se uma reavaliação fonológica, a fim de comparar a evolução terapêutica e a eficácia dos alvos escolhidos para cada sujeito. Compararam-se os inventários fonológicos e o número de traços distintivos alterados pré e pós-terapia em cada sujeito, bem como os traços distintivos enfocados na terapia pelos sons-alvo. Os sujeitos que acrescentaram mais fonemas em seus inventários fonológicos foram os que mais diminuíram o número de traços distintivos alterados. Pode-se concluir que o Modelo de Oposições Múltiplas proporcionou aquisições de fonemas no inventário fonológico, sendo esses relacionados às características dos alvos selecionados para cada sujeito. Os sujeitos cujos sons-alvo contemplavam o trabalho com a maioria dos traços alterados e com sons mais complexos na hierarquia apresentaram maiores aquisições em seus inventários fonológicos.
\end{abstract}

Descritores: Fala; Distúrbios da Fala; Fonoterapia; Criança

\section{INTRODUÇÃO}

A segmentação dos sons em traços independentes implica que quando uma criança apaga um segmento não ocorre, necessariamente, o desaparecimento de todos os traços que o compõem ${ }^{(1)}$.

A Fonologia Autossegmental propõe uma hierarquia entre os traços, passando a analisar os segmentos em camadas, o que contribuiu para os estudos da aquisição fonológica normal ou com desvios. Com base nessa teoria de traços, foi proposta

Trabalho realizado na Universidade Federal de Santa Maria - UFSM - Santa Maria (RS), Brasil, com bolsa de mestrado concedida pela Coordenação de Aperfeiçoamento de Pessoal de Nível Superior - CAPES.

(1) Mestre, Residente do Programa de Residência Multiprofissional Integrada em Sistema Público de Saúde da Universidade Federal de Santa Maria - UFSM

- Santa Maria (RS), Brasil.

(2) Doutora, Professora do Curso de Graduação em Fonoaudiologia e do Programa de Pós-Graduação em Distúrbios da Comunicação Humana da Universidade Federal de Santa Maria - UFSM - Santa Maria (RS), Brasil.

(3) Doutora, Professora do Curso de Graduação em Letras e dos Programas de Pós-Graduação em Letras e em Distúrbios da Comunicação Humana da Universidade Federal de Santa Maria - UFSM - Santa Maria (RS), Brasil.

Endereço para correspondência: Marizete Ilha Ceron. R.Bentevi, 215, Bairro JK, Santa Maria (RS), Brasil, CEP: 97035-130. E-mail: marizeteceron@ hotmail.com

Recebido em: 14/4/2009; Aceito em: 7/7/2009 uma hierarquia para o português, o Modelo Implicacional de Complexidade de Traços (MICT) ${ }^{(2)}$, como pode ser observado na Figura 1.

O MICT prevê as possibilidades da aquisição segmental sob a forma de caminhos a serem percorridos durante a aquisição. A partir do estado zero de complexidade (E0), partem caminhos (rotas) levando aos traços marcados e às combinações de traços. Quanto maior a distância do ponto zero, mais complexos são esses caminhos. Se, em um mesmo caminho, existem combinações de traços, significa que, entre eles, há uma relação de implicação.

Os primeiros traços marcados a serem especificados seriam o [-ant], que faz com que se estabeleça o/n/; o [+voz], levando à representação do /b/ e/ou /d/; e o [dors], levando à representação do $/ \mathrm{k} /$. Esses traços não são representados simultaneamente, um deles é especificado primeiro. Uma vez especificado o traço [dors], a criança pode especificar o traço [+voz], levando à produção do /b/ou /d/. A combinação destes dois traços marcados [dors, +voz] só vai acontecer após ambos já terem sido especificados individualmente nas estruturas menos complexas, isto é, o /g/ vai surgir no inventário fonológico se antes já houver um /k/ e um /b/ e/ou /d/. Uma vez especificado o traço [+voz], a criança pode especificar o traço [+cont], levando à representação das fricativas labiais 


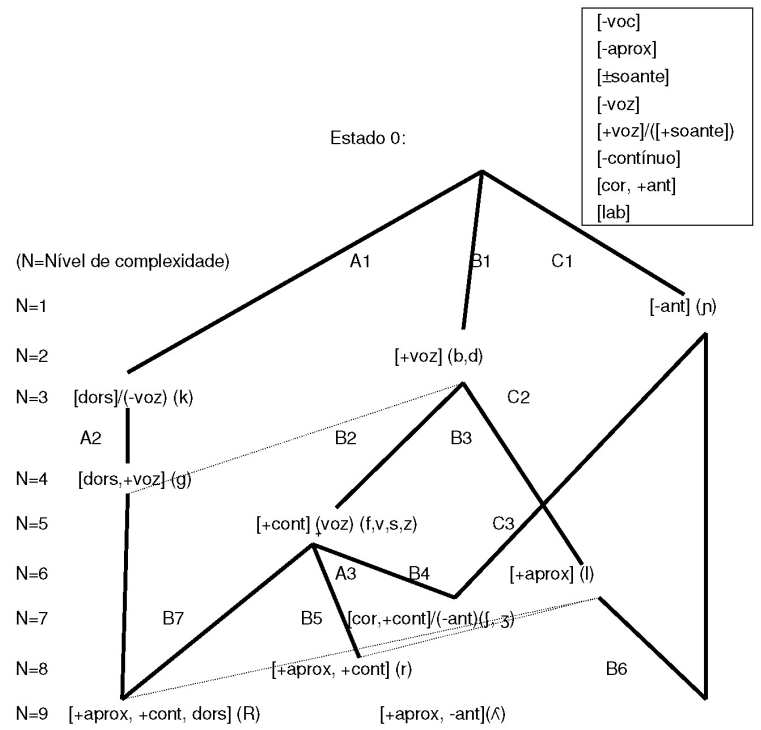

Fonte: Mota HB. Aquisição segmental do português: um modelo implicacional de complexidadede traços [Tese]. Porto Alegre: Faculdade de Letras. Pontifícia Universidade Católica do Rio Grande do Sul; 1996.

Figura 1. Modelo Implicacional de Complexidade de Traços (MICT), proposto por Mota (1996, p.154).

e coronais [+ant] (/f/ e/ou /v/ e/ou /s/ e/ou /z/) e/ou o traço [+aprox], levando à representação do /l/. Depois de especificar o traço [+cont] e já ter especificado o [-ant] para as nasais, a criança pode especificar esses dois traços levando à representação do /S/ e/ou /3/. A combinação de traços [+aprox, +cont] para a representação do /r/ depende da especificação do [+cont] e, geralmente, da presença do [+aprox], embora esta última relação implicacional seja mais fraca. A combinação de traços [+aprox, -ant], é necessária para a representação do / $/ /$, ou seja, o inventário terá $/ \mathrm{K} / \mathrm{se}$ antes tiver $/ \mathrm{l} / \mathrm{e} / \mathrm{n} /$.

A combinação de traços [+aprox, +cont, dors] que compõem o /R/ mantém relação de implicação com a combinação de traços [dors, +voz], com o traço [+cont] e, na maior parte das vezes, com o traço [+aprox], embora esta seja uma relação implicacional mais fraca. Assim, um inventário terá /R/, se já tiver $/ \mathrm{g} /$ (e consequentemente $/ \mathrm{k} /$ ), pelo menos, uma fricativa labial ou coronal e, em geral, a líquida /1/.

Os traços estão dispostos em níveis crescentes de complexidade entre os traços marcados. Na hierarquia, o primeiro traço a ser especificado é o [-ant] (/n/), que tem a menor complexidade (N1), seguido pelo traço [+voz], levando à representação de /b/ e/ou /d/, que está no segundo nível (N2) e do traço [dors] (/k/), que está no nível três (N3). No nível quatro (N4), encontra-se a combinação de traços [dors, +voz], na qual irá surgir o/g/. Após especificar o traço [+voz], a criança pode especificar o traço [+cont] e/ou o [+aprox] que correspondem, respectivamente, aos níveis cinco (N5) e seis (N6) de complexidade. No N5, encontram-se /f/, /v/, /s/ e /z/ e no N6 /l/. No nível sete (N7), estão a combinação [cor, -ant $] /[+$ cont $](/ / /$ e $/ 3 /)$. No nível oito (N8), a combinação de traços [+aprox, +cont] (/r/) e, por fim, as combinações de traços marcados [+aprox, -ant] $(/ \mathcal{K} /)$ e [+aprox, +cont, dors] (/R/), que correspondem ao maior nível de complexidade (N9).
O MICT passou a ser utilizado para auxiliar na escolha dos alvos para a terapia fonológica. A terapia fundamentada na fonologia busca a reorganização do inventário de sons da criança e a capacidade de generalização ${ }^{(3-7)}$. Num estudo ${ }^{(8)}$ é relatado que o processo terapêutico, baseado na hierarquia de traços, facilitou o processo de generalização de traços comprometidos, acelerando a evolução da terapia.

Em outro trabalho ${ }^{(9)}$ foi referido que o tratamento de propriedades mais complexas no inventário fonológico resulta em maiores ganhos fonológicos.

O Modelo de Oposições Múltiplas ${ }^{(10,11)}$ foi desenvolvido como um modelo alternativo para crianças falantes do inglês com severos desvios de fala. Esse modelo tem como objetivo induzir divisões fonêmicas múltiplas, previamente desativadas, com a finalidade de reduzir os homônimos no inventário fonológico da criança.

Um estudo ${ }^{(10)}$ mostrou a aplicação desse modelo em uma criança com desvio fonológico, sendo possível verificar que o mesmo proporcionou uma sistemática expansão do inventário fonológico na ausência de contrastes fonêmicos.

Crianças com desvio fonológico, em geral os mais graves, apresentam perda da função contrastiva de vários sons, isto é, substituem diversos fonemas por um único ${ }^{(12)}$. Quando dois ou mais sons são produzidos da mesma maneira, mas têm significados diferentes; há quebras na comunicação, reduzindo a inteligibilidade de fala ${ }^{(10)}$.

No Modelo de Oposições Múltiplas, são contrastados todos os sons-alvo (diversos fonemas produzidos por um único) com o que é substituído. Por exemplo, no inglês, uma criança que substitui os sons /s, $\int, \mathrm{k}, \mathrm{t} J /$ por $[\mathrm{t}] \mathrm{em}$ seu inventário teria como alvos de tratamento o som substituto [t] e os substituídos $\left.\left[\mathrm{s}, \int, \mathrm{k}, \mathrm{t}\right]\right]$ simultaneamente ${ }^{(10)}$. $\mathrm{O}$ fonema substituto indica que a criança desenvolveu uma estratégia para acomodar um inventário limitado de sons ${ }^{(10,12)}$. Uma autora ${ }^{(12)}$ refere que esta estratégia, de substituir vários sons por um único, está ligada diretamente à seleção dos sons-alvo de terapia nesse modelo.

A escolha dos fonemas-alvo para tratamento é um dos aspectos da intervenção fonológica que pode ser programado para facilitar as mudanças no inventário de sons. Os sons-alvo selecionados têm como objetivo induzir as maiores mudanças fonológicas e a generalização no inventário de sons ${ }^{(9)}$. Outra autora $^{(13)}$ sugere que as palavras-alvo não podem ser consideradas como fatores definidores da evolução do tratamento, mas, sim, favorecedoras às mudanças na terapia.

O objetivo deste estudo foi verificar a eficácia da proposta de terapia de Oposições Múltiplas, considerando a escolha dos sons-alvo, e analisar a aquisição de fonemas no inventário fonológico e os traços distintivos, enfocados a partir dos sons-alvo.

\section{APRESENTAÇÃO DOS CASOS CLÍNICOS}

Esta pesquisa foi aprovada e registrada no Gabinete de Projetos (GAP) sob $\mathrm{n}^{\circ} 018278$ e pelo Comitê de Ética em Pesquisa (CEP) sob n ${ }^{\circ}$ 108/05, da Universidade Federal de Santa Maria (UFSM).

Os pais e/ou responsáveis pelas crianças, envolvidas neste estudo, receberam esclarecimentos sobre a pesquisa e 
assinaram o Termo de Consentimento Livre e Esclarecido, permitindo a participação dos filhos e posterior publicação dos resultados em meios acadêmicos.

Para constituir o grupo pesquisado, conforme os critérios de inclusão nesta pesquisa, as crianças deveriam possuir: diagnóstico de desvio fonológico; alteração fonológica compatível com a proposta da autora ${ }^{(10)}$ do modelo, para a escolha dos alvos de terapia, ou seja, substituir muitos sons para um som; apresentar audição normal; e não apresentar alterações significativas em avaliações fonoaudiológicas e complementares realizadas, à exceção da avaliação fonológica.

Para descartar comprometimentos que pudessem interferir no desenvolvimento da linguagem, os sujeitos foram submetidos às avaliações: anamnese, linguagem compreensiva e expressiva, sistema estomatognático, exame articulatório, discriminação auditiva, consciência fonológica, processamento auditivo simplificado e avaliação fonológica. Foram ainda, submetidos à inspeção do meato acústico externo, avaliação auditiva e neurológica. Se os resultados da inspeção do meato acústico externo e/ou da audiometria não estivessem dentro dos padrões de normalidade, os sujeitos seriam encaminhados à avaliação otorrinolaringológica.

A partir disso, foram selecionadas para o estudo cinco crianças, sendo três do gênero feminino e duas do gênero masculino, com idades variando entre 4:2 e 8:11.

A coleta dos dados de fala foi feita através do instrumento Avaliação Fonológica da Criança (AFC) ${ }^{(14)}$. Após, foram realizadas as análises contrastiva e por traços distintivos. Considerou-se um fonema adquirido quando este ocorreu de $80 \%$ a $100 \%$ das vezes; parcialmente adquirido, de $40 \%$ a 79\%; e não adquirido, de $0 \%$ a $39 \%$ das possibilidades ${ }^{(15)}$.

A análise por traços distintivos teve como objetivo observar quais foram as maiores dificuldades relacionadas a estes, quais seriam os traços enfocados em terapia e quais as mudanças obtidas no inventário fonológico de cada sujeito. Essa análise foi realizada a partir das substituições, observadas na análise contrastiva. Consideraram-se todas as alterações de traços que ocorreram em mais de $10 \%$ das possibilidades. Optou-se por esse percentual por julgar que, acima dele, as substituições ocorrem com maior frequência na fala, possibilitando verificar melhor as mudanças no decorrer da terapia. Para a realização desta análise, utilizou-se a Matriz Fonológica dos Segmentos Consonantais do Português ${ }^{(2)}$.

A partir do inventário fonológico, foram escolhidos os sons-alvo de tratamento, de acordo com a proposta do Modelo de Oposições Múltiplas, ou seja, foram contrastados os sons substituídos com o substituto, formando pares contrastivos. Esse procedimento foi realizado para todos os sujeitos da pesquisa. Para todos os sujeitos, os alvos foram trabalhados na posição de onset medial, ou pela inexistência dos sonsalvo na posição inicial ou dificuldade de encontrar pares de palavras na mesma.

Em relação aos sons-alvo, estes foram escolhidos de acordo com a proposta do modelo e com base no inventário fonológico de cada sujeito, por isso não foram utilizados os mesmos alvos para todos os sujeitos da pesquisa. O número de sons-alvo foi diferente, sendo dois sujeitos tratados com cinco alvos, e três, com quatro alvos. Pode-se observar que, para alguns sujeitos ( $\mathrm{S} 1, \mathrm{~S} 2$ e S3), os sons-alvo contemplavam as maiores dificuldades em termos de traços e para outros (S4 e S5), não.

Os alvos selecionados para $\mathrm{S} 1$ foram /l/, /R/, /z/ e /v/ e seu substituto [j]. As palavras-alvo, utilizadas para o tratamento, foram ['kaju], ['kalu], ['kaRu], ['kazu] e ['kavu]. Para S2 e S5, foi contrastado o [ $] \mathrm{com} / \mathrm{s} /, / \mathrm{z} / \mathrm{e} / 3 /$, cujos alvos selecionados foram ['kaJa], ['kasa], ['kaza] e ['kaza]. S3 foi tratado com /3/, /l/, / / / e /r/, mais o fonema [z] (substituto). As palavras escolhidas foram ['kaza], ['kala], ['kaКa], ['kara], ['kaza]. Os sons-alvo para $\mathrm{S} 4$ foram /z/, / / e /3/, os quais eram produzidos como [s], que também foi incluído nos alvos de tratamento. As palavras selecionadas foram ['kaJa], ['kasa], ['kaza] e ['kaza]. Esses alvos foram selecionados, porque eram os únicos sons possíveis, de acordo com a proposta da autora e o inventário fonológico de cada sujeito.

Os sujeitos foram submetidos a 25 sessões de fonoterapia pelo Modelo de Oposições Múltiplas ${ }^{(9)}$, com exceção do S3 que foi submetido a apenas 15 sessões de terapia, nas quais os alvos foram adquiridos e não houve possibilidade de continuar o tratamento com esse modelo de terapia pela incompatibilidade de escolha dos novos alvos. Após o tratamento, os sujeitos foram reavaliados (avaliação fonológica final).

A seguir, foram analisados e comparados os inventários fonológicos (fonemas e traços distintivos), alterados pré e pós-tratamento em cada sujeito, bem como os alvos utilizados durante a terapia.

Na Tabela 1, são apresentados os fonemas adquiridos e parcialmente adquiridos nos inventários fonológicos dos cinco sujeitos pré e pós-tratamento.

Nota-se que a maioria dos sujeitos adquiriu fonemas em seus inventários fonológicos. É possível observar que S2 e S3 foram os que mais adquiriam fonemas (oito), seguido pelo S1 (cinco fonemas) e S5 (dois fonemas). S4 não apresentou aumento no número de fonemas adquiridos, porém os fonemas $/ \mathrm{v} /$ e /z/, ausentes na avaliação inicial, tornaram-se parcialmente adquiridos após a terapia. Ainda, todos os sujeitos apresentavam dificuldades com as classes das plosivas, fricativas e líquidas e S2 apresentava, também, problemas com as nasais.

A Tabela 2 apresenta os traços distintivos alterados pré e pós-terapia e os sons-alvo, selecionados para o tratamento de cada sujeito.

Verificou-se que S1 apresentava um total de 11 traços distintivos alterados com 38 ocorrências; S2, nove com 36 ocorrências; S3 e S4, sete traços distintivos alterados com 24 e 20 ocorrências, respectivamente; e S5, seis traços distintivos alterados com 19 ocorrências. Desses, os que mais apresentaram progressos, em relação ao número de ocorrência de traços distintivos alterados, foram S2 e S3, que diminuíram de 36 para 20 e de 24 para cinco, respectivamente.

S1 e S3 eliminaram, após o tratamento, três oposições de traços distintivos alterados. S1 apresentava alteração em 11 traços distintivos, sendo que foram eliminados, na avaliação final, os traços [-voz], [+cont] e [labial]. S3 eliminou as alterações nos traços [+soante], [+voz] e [-cont] dos sete que estavam alterados na avaliação inicial. S2, S4 e S5 tiveram apenas um traço distintivo alterado, eliminado após a terapia, sendo respectivamente o [+soante], para S2; o [-cont], para S4; e o [cor,-ant], para S5. Nesta perspectiva, S1 e S3 tiveram 
Tabela 1. Fonemas adquiridos e parcialmente adquiridos no inventário fonológico dos sujeitos

\begin{tabular}{|c|c|c|c|}
\hline \multirow[t]{2}{*}{ Sujeitos } & Avaliação & Inventário fonológico & $\mathrm{N}^{\circ}$ de fonemas adquiridos \\
\hline & \multicolumn{3}{|c|}{$\mathrm{p}, \mathrm{b}, \mathrm{t}, \mathrm{d}, \mathrm{k}, \mathrm{g}, \mathrm{f}, \mathrm{v}, \mathrm{s}, \mathrm{z}, \int, 3, \mathrm{~m}, \mathrm{n}, \mathrm{n}, \mathrm{l}, \mathrm{r}, \kappa, \mathrm{R}$} \\
\hline \multirow[t]{2}{*}{ S1 } & $\mathrm{Al}$ & $p, b, t,(d)^{*}, k, m, n,(n)^{*}$ & 6 \\
\hline & AF & $\mathrm{p},(\mathrm{b})^{*}, \mathrm{t}, \mathbf{d}, \mathrm{k}, \mathbf{v}, \mathbf{s},(\mathrm{z})^{*}, \int, \mathrm{m}, \mathrm{n}, \mathbf{n},(\mathrm{R})^{*}$ & 10 \\
\hline \multirow[t]{2}{*}{ S2 } & Al & $\mathrm{p},(\mathrm{b})^{\star}, \mathrm{t},(\mathrm{d})^{\star},(\mathrm{k})^{\star},(\mathrm{f})^{\star}, \mathrm{v},\left(\int\right)^{\star},(\mathrm{m})^{\star},(\mathrm{n})^{\star},(\mathrm{n})^{\star}$ & 3 \\
\hline & AF & $\mathrm{p}, \mathbf{b}, \mathbf{t}, \mathbf{d},(\mathrm{k})^{\star},(\mathrm{g})^{\star}, \mathbf{f}, \mathrm{v}, \mathbf{\int}, \mathbf{3}, \mathbf{m}, \mathbf{n}, \mathbf{n},(\mathrm{l})^{\star},(\mathrm{R})^{\star}$ & 11 \\
\hline \multirow[t]{2}{*}{ S3 } & Al & $\mathrm{p},(\mathrm{b})^{\star}, \mathrm{t}, \mathrm{d}, \mathrm{k}, \mathrm{f}, \mathrm{s},(\mathrm{z})^{\star},\left(\int\right)^{\star}, \mathrm{m}, \mathrm{n}, \mathrm{n}, \mathrm{R}$ & 10 \\
\hline & AF & $\mathrm{p}, \mathbf{b}, \mathrm{t}, \mathrm{d}, \mathrm{k}, \mathbf{g}, \mathrm{f}, \mathbf{v}, \mathrm{s}, \mathbf{z}, \int, \mathbf{z}, \mathrm{m}, \mathrm{n}, \mathrm{n}, \mathbf{I}, \boldsymbol{\kappa}, \mathbf{R}$ & 18 \\
\hline \multirow[t]{2}{*}{ S4 } & $\mathrm{Al}$ & $p, t, k, f, s, m, n, n, l$ & 9 \\
\hline & AF & $p, t, k, f,(v)^{*}, s,(z)^{*}, m, n, n, I$ & 9 \\
\hline \multirow[t]{2}{*}{ S5 } & Al & $p,(b)^{*}, t,(d)^{*}, k, f,(v)^{*}, s, \int, m, n, n,(I)^{*}, R$ & 10 \\
\hline & AF & $\mathrm{p},(\mathrm{b})^{\star}, \mathrm{t}, \mathbf{d}, \mathrm{k}, \mathrm{f},(\mathrm{v})^{\star}, \mathrm{s},(\mathrm{z})^{\star}, \int, \mathbf{3}, \mathrm{m}, \mathrm{n}, \mathrm{n},(\mathrm{l})^{\star}, \mathrm{R}$ & 12 \\
\hline
\end{tabular}

Legenda: $\mathrm{Al}$ = avaliação inicial; $\mathrm{AF}=$ avaliação final; $\left(\right.$ ) ${ }^{\star}=$ fonema parcialmente adquirido; Negrito = fonemas adquiridos após terapia

Tabela 2. Traços distintivos alterados pré e pós-tratamento e os sons-alvo selecionados para terapia

\begin{tabular}{|c|c|c|c|c|c|c|c|c|c|c|}
\hline \multirow{3}{*}{ Traços distintivos alterados } & \multicolumn{10}{|c|}{ Sujeitos } \\
\hline & \multicolumn{2}{|c|}{ S1 } & \multicolumn{2}{|c|}{ S2 } & \multicolumn{2}{|c|}{ S3 } & \multicolumn{2}{|c|}{ S4 } & \multicolumn{2}{|c|}{ S5 } \\
\hline & $\mathrm{Al}$ & AF & $\mathrm{Al}$ & AF & $\mathrm{Al}$ & AF & Al & AF & $\mathrm{Al}$ & $\mathrm{AF}$ \\
\hline [+soante] $\rightarrow[$-soante] & * & * & 1 & 0 & 4 & 0 & * & * & * & * \\
\hline$[$-soante $] \rightarrow[+$ soante $]$ & 4 & 2 & * & * & * & * & * & * & * & * \\
\hline$[+$ aprox $] \rightarrow[$-aprox $]$ & 5 & 4 & 6 & 3 & 4 & 1 & 2 & 1 & 4 & 3 \\
\hline$[$-cons $] \rightarrow[+$ cons $]$ & 9 & 7 & 5 & 3 & 1 & 1 & 2 & 1 & 4 & 3 \\
\hline$[+\mathrm{voz}] \rightarrow[-\mathrm{voz}]$ & 3 & 5 & 4 & 1 & 8 & 0 & 8 & 8 & 4 & 7 \\
\hline$[-\mathrm{voz}] \rightarrow[+\mathrm{voz}]$ & 1 & 0 & * & * & * & * & * & * & * & * \\
\hline$[+$ cont $] \rightarrow[-$ cont $]$ & 2 & 0 & 1 & 1 & 0 & 1 & 1 & 1 & * & * \\
\hline$[$-cont $] \rightarrow[+$ cont $]$ & 2 & 3 & 3 & 2 & 3 & 0 & 1 & 0 & 2 & 1 \\
\hline [labial] $\rightarrow$ [cor] & 1 & 0 & * & * & * & * & * & * & * & * \\
\hline$[\mathrm{cor}$, +ant $] \rightarrow[$ cor, -ant $]$ & 8 & 6 & 9 & 6 & 1 & 1 & 2 & 1 & 4 & 4 \\
\hline$[$ cor, -ant $] \rightarrow[$ cor, +ant $]$ & 2 & 2 & 6 & 3 & 3 & 1 & 4 & 5 & 1 & 0 \\
\hline [dors] $\rightarrow$ [cor, -ant] & 1 & 1 & 1 & 1 & * & * & * & * & * & * \\
\hline Total & 38 & 30 & 36 & 20 & 24 & 5 & 20 & 17 & 19 & 18 \\
\hline Sons-alvo & $/ \mathrm{I}, \mathrm{R}, \mathrm{v}, \mathrm{s}, \mathrm{j} /$ & & $/ s, z, \int, 3 /$ & & $|z, I, \Lambda, r, z|$ & & $/ s, z, \int, 3 /$ & & $/ s, z, \int, 3 /$ & \\
\hline
\end{tabular}

Legenda: $\mathrm{Al}=$ avaliação inicial; $\mathrm{AF}=$ avaliação final

* Não houve ocorrência de alteração destes traços distintivos

um melhor desempenho em relação à aquisição de traços. É interessante destacar que foram esses os únicos sujeitos que foram tratados com líquidas e fricativas.

Também cabe salientar que o traço que mais sofreu regressão, ou seja, apresentou aumento de ocorrência de alteração após o tratamento, foi o [+voz] no tratamento de S1 e S5.

Os sujeitos (S2, S3) que mais fonemas acrescentaram em seus inventários fonológicos foram, também, os que mais diminuíram o número de traços distintivos alterados.

Na Tabela 3, são apresentados os resultados da análise estatística, comparando as médias dos sons presentes nas avaliações inicial e final, em relação ao inventário fonológico e traços distintivos alterados.

Observou-se que houve aumento do número de fonemas adquiridos no inventário fonológico e uma redução do número dos traços distintivos alterados após o tratamento, sendo verificada uma diferença estatisticamente significativa entre as avaliações inicial e final para os sujeitos pesquisados.
Tabela 3. Média dos sons presentes no inventário fonético, fonemas adquiridos no inventário fonológico geral e traços distintivos alterados pré e pós-tratamento

\begin{tabular}{lccc}
\hline & Al & AF & Valor de p \\
& Média (DP) & Média (DP) & \\
\hline IF & $7,2(2,7)$ & $11,8(3,6)$ & $0,03^{*}$ \\
TDA & $27,4(9)$ & $18(9)$ & $0,03^{*}$ \\
\hline
\end{tabular}

*Valores estatisticamente significantes $(p<0,05)$

Legenda: IF = inventário fonológico; TDA = traços distintivos alterados; $\mathrm{Al}=$ avaliação inicial; AF = avaliação final; DP = desvio-padrão

\section{DISCUSSÃO}

S1 apresentou alteração em vários traços distintivos, sendo os traços [+cons], [cor/+ant], [+aprox] e [-soante] os que apresentavam as maiores dificuldades. Os alvos escolhidos, conforme a proposta da autora ${ }^{(10)}$, foram /l/, /R/, /v/, /s/, [j]. O 
enfoque desses sons possibilitou o contraste dos traços [cor] x [lab] x [dors], [+aprox] x [-aprox] e [+soante] x [-soante]. Observou-se que as maiores dificuldades em termos de traços estavam presentes nos fonemas tratados. Após o tratamento, notou-se diminuição dos traços distintivos alterados e a aquisição de cinco novos fonemas /d/, /v/, /s/, / /, / $/$ no inventário fonológico.

Para S1, em relação aos níveis de complexidades, a partir dos alvos estimulados, era esperada a aquisição de $/ \mathrm{n} /($ rota* C1, Nível 1), /g/ (A2, N4), /f, v, s, z/ (B2, N5) e /d/ (B1, N2). Todos esses segmentos situam-se em níveis inferiores ao do som-alvo. As aquisições de /n/, /d/, /v/ e /s/ foram previstas pelo $\mathrm{MICT}^{(2)}$, enquanto /S/, não. Resultados semelhantes foram encontrados em outro estudo $^{(6)}$, ao analisarem a generalização implicacional. Alguns estudos ${ }^{(8,9)}$ afirmam que o tratamento de propriedades mais complexas no inventário fonológico resulta em maiores mudanças.

Os alvos escolhidos para S1 previam a aquisição de vários sons de níveis menos complexos que os dos sons-alvo. Considerando a hierarquia, alguns dos alvos pertenciam a níveis mais complexos, como é o caso do alvo pertencente à classe das líquidas (R).

O S2 apresentou, na avaliação inicial, dificuldades principalmente com o traço [cor/₫ant]. O traço [+aprox] apresentou a mesma quantidade de alterações do traço [cor/-ant]. Este sujeito foi estimulado com os alvos /s/, /z/, / / /, /3/, todos contendo o traço [cor], sendo dois [cor/+ant] e dois [cor/-ant]. Com o tratamento, os traços, com maiores dificuldades para o sujeito, foram contrastados ou reforçados pela estimulação com os alvos. Após, percebeu-se a diminuição desses e de outros traços que estavam alterados na avaliação inicial.

A partir do tratamento de $\mathrm{S} 2$, espera-se que os alvos /s, z/ que apresentam os traços [+cont] $( \pm \mathrm{voz})$ e $/ \int, 3 /$ e que têm os traços mais marcados [cor,+cont]/(-ant) favoreçam a aquisição de segmentos como /p, t, m, n/ (E0), /b,d/ (B1,N2), /f, v, s, z/ $(\mathrm{B} 2, \mathrm{~N} 5)$ e $/ \mathrm{n} /(\mathrm{C} 1, \mathrm{~N} 1)$, considerando que todos pertencem a níveis inferiores aos dos sons-alvo. Observou-se que S2 percorreu alguns caminhos possíveis e necessários, para especificar os traços que apresentavam dificuldade. Este foi um dos sujeitos que mais adquiriu fonemas no inventário fonológico, são eles: /b, d, f, J, 3, m, n, n/. A aquisição desses fonemas, a partir dos alvos tratados, concordou com as previsões do $\mathrm{MICT}^{(2)}$. Outra autora $^{(9)}$ enfatizou que as maiores mudanças são induzidas por tratamentos de sons mais complexos. O tratamento com as fricativas (sons mais marcados) possibilitou a aquisição de plosivas (sons menos marcados), esse achado concorda com outro estudo ${ }^{(3)}$.

Os alvos fricativos (pertencentes aos níveis 5 e 7 do MICT) foram ótimas opções para o tratamento de S2, visto que havia alterações até mesmo no E0 (de menor complexidade). Assim, para esse sujeito, havia vários caminhos a percorrer na aquisição de sons, ao contrário de S4 e S5, que foram tratados com os mesmos alvos, porém, analisando o inventário de cada um, eles não apresentavam muitas possibilidades de aquisições de sons.

S3 foi o sujeito que mais diminuiu o número de traços distintivos alterados. A principal dificuldade se encontrava no traço [+voz]. Todos os fonemas, escolhidos para o tratamento, apresentavam esse traço $(/ 3,1, \kappa, \mathrm{r}, \mathrm{z} /)$. Na avaliação final, observou-se a extinção desse e de outros traços alterados na avaliação inicial, como o traço [+soante] e o [-cont] (traços contrastados nos sons-alvo de terapia). Foi o outro sujeito que mais adquiriu fonemas no inventário fonológico, isto é, aquisição de /b, g, v, z, $\int, 3,1, \kappa /$, todos apresentando o traço [+voz]. Um estudo ${ }^{(3)}$ refere que o trabalho com um membro marcado de um par cognato leva à aquisição do membro não marcado, como é o caso da estimulação de /3/ que auxiliou a aquisição do / $/$ nesse sujeito.

O tratamento com /3/ que apresenta os traços $[\mathrm{cor},+$ cont $] /$ (-ant) pela relação implicacional direta, pode favorecer a aquisição de /v, z/ (B2, N5). O /1/, com o traço [+aprox], implicaria a aquisição de /b/ (B1,N2); o / / / que tem os traços [+aprox, -ant] beneficia a aquisição de /1/ $(\mathrm{B} 3, \mathrm{~N} 6)$ e de todos os outros níveis abaixo dos alvos, como o/g/ (A2, N4). Foram adquiridos todos os fonemas previstos pelo MICT, a partir dos alvos estimulados. Estudos ${ }^{(2,8)}$ observaram que a estimulação de um traço, hierarquicamente mais complexo, possibilitou a aquisição de traços menos complexos, bem como a generalização do próprio traço para outros fonemas não trabalhados.

Assim como para S1, S3 apresentava como alvos sons fricativos e líquidos, o que possibilitou mais caminhos a percorrer, visto que sons de níveis mais complexos estavam sendo tratados.

Tal como S3, S4 apresentou maior dificuldade com o traço [+voz], o qual não diminuiu após o tratamento. Apenas dois, dos quatro alvos, apresentavam o traço [+voz]. O enfoque desses sons possibilitou o contraste dos traços [+voz] x [-voz]. Esse sujeito foi o único que não adquiriu nenhum fonema no inventário fonológico.

Analisando as previsões do MICT para S4, esperava-se que os alvos /s, z/ (B2, N5), que apresentam os traços [+cont] ( \pm voz), pudessem favorecer a aquisição de $/ \mathrm{b} / \mathrm{e} / \mathrm{d} /$ pela relação implicacional direta e os fonemas / $\int, 3 /(B 5, N 7)$, que têm os traços [cor,+cont $] /(-a n t)$, proporcionassem generalização para /v/ e/z/ (B2,N5). Não se observou generalização para nenhuma das plosivas, apenas para as fricativas que se tornaram parcialmente adquiridas no inventário fonológico. Esse achado não está de acordo com outro estudo ${ }^{(3)}$, em que a estimulação com fricativas levou à aquisição de plosivas.

Os sons selecionados para S4 de acordo com o MICT não possibilitavam muitos caminhos a percorrer, apenas de /b, d/ e /f, v/. Para esse sujeito, a inclusão de uma líquida nos alvos de tratamento poderia ter contribuído para uma melhor evolução na terapia. Porém, conforme a proposta do modelo, isso não era possível.

S5 foi o sujeito que menos diminuiu o número de traços distintivos alterados. Ressalta-se que as maiores dificuldades estavam nos traços [+aprox], [+voz] e [cor/+ant]. Dos alvos enfocados em terapia, nenhum apresentava o traço [+aprox], dois contrastavam o [+voz] x [-voz] e dois, [cor/+ant] x [cor/-ant].

Os alvos /s, z/ (B2, N5), apresentando os traços [+cont]

\footnotetext{
* Rota: diferentes caminhos percorridos pelas crianças durante a aquisição fonológica.
} 
( \pm Voz), poderiam favorecer a aquisição de todos os níveis abaixo dele, principalmente /b, d/, considerando a relação implicacional direta. Achados parecidos foram relatados por outras autoras $^{(6)}$, ao analisarem sujeitos com diferentes gravidades dos desvios fonológicos.

Os segmentos $/ \int, 3 /$, que têm os traços [cor,+cont]/(-ant), implicam a aquisição de quaisquer outras fricativas $(v, z)$ que têm o traço [+cont]. Esse sujeito acrescentou apenas dois fonemas (/d/ e /3/) no inventário fonológico. Concordando com achados anteriores ${ }^{(3)}$, que constataram que o trabalho com um som mais marcado, no caso as fricativas, este levou à aquisição de sons menos marcados, como é o caso da plosiva /d/ para o S5.

Considerando os sons-alvo escolhidos pelas previsões do MICT, esse sujeito tinha poucos caminhos a percorrer, visto que /p, t, m, n/ (E0), /f, s/ (B2,N5) e /n/ (C1,N1) estavam adquiridos; /b, d/ (B1,N2) e/v/ (B2,N5), parcialmente adquiridos; e /z/ (B2,N5) ausente na avaliação inicial. Foi observada a generalização para /d/ e /3/. Para esse sujeito, considerando a hierarquia dos traços distintivos para as escolhas dos alvos, talvez fosse melhor optar por alvos em níveis mais complexos na hierarquia, possibilitando maiores generalizações. Sons mais complexos são caracterizados como aqueles de aquisição mais tardia e esses causam maiores generalizações ${ }^{(9)}$. Outros autores $^{(8)}$ referem que a hierarquia de traços facilitou o pro- cesso de generalização de traços comprometidos, acelerando a evolução da terapia.

Em relação aos traços distintivos, todos os sujeitos apresentavam alteração nos traços [+voz], [+aproximante] e [coronal/t_anterior]. Os sujeitos cujos sons-alvo contemplavam o trabalho com a maioria desses traços alterados apresentaram maiores aquisições em seus inventários fonológicos. Com isso, sugere-se a relevância de analisar, além do inventário fonológico de cada sujeito, os traços distintivos alterados para a escolha dos sons-alvo de terapia. Isso poderá tornar o tratamento mais rápido e eficaz. Um estudo ${ }^{(13)}$ mostrou que as palavras-alvo selecionadas para serem usadas em terapia são favorecedoras na organização dos inventários fonológicos dos sujeitos.

\section{CONSIDERAÇÕES FINAIS}

Os achados desta pesquisa permitem concluir que o Modelo de Oposições Múltiplas proporcionou aquisições de fonemas no inventário fonológico, sendo estes relacionados às características dos alvos, selecionados para cada sujeito. Além disso, os sujeitos, cujos sons-alvo contemplavam o trabalho com a maioria dos traços alterados e com sons mais complexos na hierarquia, apresentaram mais aquisições em seus inventários fonológicos.

\begin{abstract}
The targets of the treatment can facilitate or difficult acquisitions in the phonological inventory. This study had the aim to verify the effectiveness of the therapy proposed by the Multiple Oppositions Model, taking into consideration the target sounds chosen, as well as to analyze the acquired sounds and the distinctive features, based on the target sounds. The researched group comprised five children with phonological disorder, with an average age of 6;1 (years;months). Speech-language pathology and complementary evaluations were carried out. After the assessments, the subjects underwent speech therapy using the Multiple Oppositions Model. After treatment, another evaluation was carried out, in order to compare the therapeutic evolution and the effectiveness of the chosen sound targets for each subject. The phonological inventory and the number of altered distinctive features before and after therapy were compared for each subject, as well as the distinctive features focused during therapy. The subjects who added more phonemes to their phonological inventory were the ones that most decreased the number of altered distinctive features. It can be concluded that the Multiple Oppositions Model provided improvements on the phonological inventory that were related to the characteristics of the target sounds selected for each subject. The subjects whose target sounds contemplated the work with the majority of the altered distinctive features and the most complex sounds in the phonological hierarchy presented greater acquisitions in their phonological inventories.
\end{abstract}

Keywords: Speech; Speech disorders; Speech therapy; Child

\title{
REFERÊNCIAS
}

1. Bisol L. Introdução aos estudos de fonologia do português brasileiro. 4a ed. Porto Alegre: EDIPUCRS; 2005.

2. Mota HB. Os padrões de erros nas alterações de fala analisados pelo modelo implicacional de complexidade de traços. Pró-Fono. 1998;10(1):73-80.

3. Mota HB, Pereira LF. A generalização na terapia dos desvios fonológicos: experiência com duas crianças. Pró-Fono. 2001;13(2):141-6.

4. Crosbie S, Holm A, Dodd B. Intervention for children with severe speech disorder: a comparison of two approaches. Int J Lang Commun Disord. 2005;40(4):467-91.

5. Mota HB, Keske-Soares M, Bagetti T, Ceron MI, Melo Filha MGC.
Análise comparativa da eficiência de três diferentes modelos de terapia fonológica. Pró-Fono. 2007;19(1):67-74.

6. Barberena LS, Keske-Soares M, Mota HB. Generalização baseada nas relações implicacionais obtida pelo modelo "ABAB-Retirada e Provas Múltiplas”. Rev Soc Bras Fonoaudiol. 2008;13(2):143-53.

7. Tyler AA. Commmentary on "treatment decisions for children with speech-sound disorder": revisiting the past in EBF. Lang Speech Hear Serv Sch. 2006;37(4):280-3.

8. Spíndola RA, Payão LMC, Bandini HHM. Abordagem fonoaudiológica em desvios fonológicos fundamentada na hierarquia dos traços distintivos e na consciência fonológica. Rev CEFAC. 2007;9(2):180-9. 
9. Gierut JA. Complexity in phonological treatment: clinical factors. Lang Speech Hear Serv Sch. 2001;32(4):229-41.

10. Williams AL. Multiple oppositions: theoretical foundations for an alternative contrastive intervention approach. Am J Speech-Lang Pathol. 2000;9(4):282-8.

11. Williams AL. A systematic perspective for assessment and intervention: a case study. Int Speech Lang Pathol. 2006;8(3):245-56.

12. Williams AL. Assessment, target selection, and intervention: dynamic interactions within a systemic perspective. Top Lang Disord. $2005 ; 25(3): 231-42$.
13. Blanco-Dutra AP. A aquisição das fricativas /f/, /v/, /s/ e /3/ por crianças com desvio fonológico [tese]. Porto Alegre: Faculdade de Letras. Pontifícia Universidade Católica do Rio Grande do Sul; 2008.

14. Yavas M, Hernandorena CLM, Lamprecht RR. Avaliação fonológica da criança: reeducação e terapia. Porto Alegre: Artes Médicas; 1991.

15. Bernhardt B. Developmental implications of nonlinear phonological theory. Clin Linguist Phon. 1992;6(4):259-81. 